\title{
Atributos químicos de Latossolo sob plantio direto adubado com esterco de bovinos e fertilizantes minerais
}

\author{
Milena Barcellos', Antonio Carlos Vargas Motta², Volnei Pauletti², \\ José Carlos Peixoto Modesto da Silva ${ }^{3}$, Julierme Zimmer Barbosa ${ }^{2}$ \\ 'Secretaria de Estado da Educação do Paraná, Santa Maria do Oeste, PR, Brasil \\ ¿Universidade Federal do Paraná, Curitiba, PR, Brasil \\ ${ }^{3}$ Universidade Federal de Viçosa, MG, Brasil \\ *Autor correspondente, e-mail: mottaufpr@gmail.com
}

\section{Resumo}

A adubação orgânica no sistema de plantio direto (SPD) vem sendo utilizada em regiões características de produção leiteira, com a finalidade de fornecer nutrientes às culturas agrícolas e possibilitar um destino à elevada produção de resíduos animais confinados. O objetivo do estudo foi avaliar os atributos químicos de um Latossolo Bruno distrófico típico em função da adubação orgânica com esterco líquido de bovinos de leite (ELBL) e com fertilizantes minerais. O experimento foi conduzido em SPD, com rotação de culturas incluindo leguminosas e gramíneas. O delineamento experimental foi em blocos casualizados em esquema fatorial com três níveis de adubação mineral $(0,50$ e $100 \%$ da dose recomendada para as culturas) e quatro níveis de adubação orgânica $(0$, 30,60 e $90 \mathrm{~m}^{3} \mathrm{ha}^{-1} \mathrm{ano}^{-1}$ ), com três repetições. Transcorridos seis anos de manejo da adubação das culturas, o solo foi coletado em cinco profundidades $(0-0,05,0,05-0,10,0,10-0,30,0,30-0,50$ e 0,50$0,80 \mathrm{~m})$. Nas amostras foram determinados os atributos químicos $\mathrm{pH}, \mathrm{H}^{+}+\mathrm{Al}^{3+}, \mathrm{Al}^{3+}, \mathrm{Ca}^{2+}, \mathrm{Mg}^{2+}, \mathrm{P}, \mathrm{K}^{+}$, condutividade elétrica (CE), saturação por bases (V), relação $\mathrm{Ca}^{2+} / \mathrm{Mg}^{2+}, \mathrm{C}$ orgânico total (COT), B, $\mathrm{Cl}, \mathrm{Fe}, \mathrm{Cu}, \mathrm{Zn}$ e Mn. A adubação mineral acidificou o solo, elevou os teores de $\mathrm{K}^{+}, \mathrm{P}$ e $\mathrm{CE}$ e, alterou os teores de Zn e Cu. A adubação orgânica com ELBL foi fonte de nutrientes ( $\mathrm{Ca}^{2+}, \mathrm{Mg}^{2+}, \mathrm{K}^{+}, \mathrm{P}, \mathrm{Zn}$ e $\mathrm{Cu})$, elevou o COT e a CE e, manteve os atributos de acidez do solo $\left(\mathrm{pH}, \mathrm{H}^{+}+\mathrm{Al}^{3+} \mathrm{e} \mathrm{V}\right)$.

Palavras-chave: macronutrientes, micronutrientes, qualidade ambiental, variabilidade vertical

\section{Chemical attributes of Oxisol in no-tillage system fertilized with manure from cattle and mineral fertilizers}

\begin{abstract}
Organic fertilization in no-tillage system (NTS) has been used in regions milk production, in an order to provide nutrients for crops and provide a destination for high production of animal waste. The aim of this study was to evaluate the chemical attributes of an Oxisol, a function of organic fertilization with liquid manure from dairy cattle (LMDC) and mineral fertilizers. The experiment was conducted under NTS with crop rotation including legumes and grasses. The treatments were distributed in three randomized blocks with factorial arrangement, using three levels of mineral fertilizer $(0,50$ and $100 \%$ of the dose recommended for the crops) and four levels of organic fertilizer $\left(0,30,60\right.$ and $90 \mathrm{~m}^{3} \mathrm{ha}$ 'year-1). After six years of fertilizer management of crops, soil was collected from five depths (0-0.05, $0.05-0.10,0.10-0.30,0.30-0.50$ e 0.50-0.80 m). The samples were determined the chemical pH, $\mathrm{H}^{+}+\mathrm{Al}^{3+}$, $\mathrm{Al}^{1+}, \mathrm{Ca}^{2+}, \mathrm{Mg}^{2+}, \mathrm{K}^{+}$, electrical conductivity $(\mathrm{EC})$, bases saturation $(\mathrm{V}), \mathrm{Ca}^{2+} / \mathrm{Mg}^{2+}$ ratio, total organic $\mathrm{C}$ (TOC), B, Cl, Fe, CU, Zn and Mn. The mineral fertilizers acidify the soil, raised the $\mathrm{K}^{+}, \mathrm{P}$ and $\mathrm{EC}$ and, changed the $\mathrm{Zn}$ and $\mathrm{Cu}$ contents. The organic fertilization with LMDC was a source of nutrients (Ca ${ }^{2+}, \mathrm{Mg}^{2+}, \mathrm{K}^{+}, \mathrm{P}, \mathrm{Zn}$ and $\left.\mathrm{CU}\right)$, raised the TOC and the EC, and kept the soil acidity attributes $\left(\mathrm{pH}, \mathrm{H}^{+}+\right.$ $\mathrm{Al}^{3+}$ and $\left.\mathrm{V}\right)$.
\end{abstract}

Keywords: macronutrients, micronutrients, vertical variability, environmental quality 


\section{Introdução}

Os solos característicos dos ambientes tropical e subtropical, em sua maioria, são originalmente ácidos e pobres em nutrientes, aspecto agravado pelo contínuo uso agrícola que resulta principalmente em redução dos teores de carbono (C) ao longo dos anos (Conceição et al., 2014). Visando a melhoria da qualidade do solo e a redução do impacto ambiental ocasionado pela atividade agrícola, têm-se adotado algumas práticas que estabelecem estratégias de utilização menos negativas, através da manutenção da matéria orgânica, manejo de resíduos vegetais e produção de matéria seca e reposição dos nutrientes exportados pelas culturas. Como principais têm-se o manejo do sistema plantio direto (SPD), rotação de culturas, adubação verde e orgânica (Nunes et al., 2011; Acosta et al., 2014).

A adubação orgânica aliada ao SPD vem sendo utilizada principalmente em regiões características de produção leiteira, com a finalidade de fornecer nutrientes às culturas agrícolas e possibilitar um destino à elevada produção de resíduos animais confinados (Mori et al., 2009). A produtividade das principais culturas de grãos tem sido elevada pela utilização de estercos na adubação, sendo que alguns aspectos relacionados com o uso destes resíduos podem ser verificados apenas em experimentos de médio e longo prazo, devido à mudança gradual das propriedades do solo(Ceretta et al., 2010; Conceição et al., 2014).

O uso contínuo de esterco em uma mesma área tende a aumentar a fertilidade do solo, especialmente dos níveis de nitrogênio (N), fósforo (P) e potássio (K) (Ceretta et al., 2010; Silva et al., 2010). Além disso, os resíduos da produção animais contêm diversos elementos, incluindo metais pesados, como zinco (Zn) e cobre (Cu), que são potencialmente acumuláveis no solo (Scherer et al., 2010). Contudo, excessos de $\mathrm{K}$ são incomuns em áreas de pecuária leiteira, principalmente quando estas são utilizadas para a produção de silagem de planta inteira, que exporta do local grande quantidade deste nutriente. Porém, ao substituir a produção de silagem pela produção de grãos, em áreas frequentemente adubadas com esterco, pode ocorrer redução expressiva da necessidade de aplicação de adubos minerais, pois parte dos nutrientes retorna ao solo pelos resíduos culturais.

Esta perspectiva aumenta com o uso do SPD, que proporciona redução da erosão, maior ciclagem de nutrientes, aumento da atividade biológica do solo e melhor aproveitamento de resíduos culturais (Ceretta et al., 2010; Conceição et al., 2014). O próprio uso desse sistema promove uma tendência inicial de aumento, seguida de estabilização das produtividades ao longo do tempo (Silva et al., 2010), principalmente em culturas leguminosas, evidenciando o aumento gradual da fertilidade do solo. Apesar do amplo uso do plantio diretono Paraná, são poucos os estudos regionais sobre aplicação de esterco nesse sistema.

Neste sentido, o objetivo do estudo foi avaliar os atributos químicos de um Latossolo Bruno distrófico típico em função da adubação orgânica com esterco líquido de bovinos de leite (ELBL) e com fertilizantes minerais.

\section{Material e Métodos}

O experimento foi conduzido na Fazenda Capão Alto, no município de Castro-PR, em área experimental da Fundação ABC (Arapoti, Batavo e Castrolanda), na região fisiográfica denominada Primeiro Planalto Paranaense, $24^{\circ}$ 44' de latitude sul e 49 55' de longitude oeste, com altitude média de $998 \mathrm{~m}$. O clima da região, segundo a classificação climática de Köppen, é do tipo $\mathrm{Cfb}$, com média anual entre $17^{\circ} \mathrm{C} \mathrm{e}$ $18^{\circ} \mathrm{C}$ e pluviosidade anual entre 1300 e $1600 \mathrm{~mm}$ (Alvares et al., 2013).

O solo da área foi classificado como Latossolo Bruno Distrófico típico A proeminente textura argilosa fase campo subtropical com relevo suave ondulado (Embrapa, 2002) com as características químicas do solo da área experimental antes da instalação do experimento e de área adjacente sob condição nativa apresentadas na Tabela 1. Anteriormente a implantação do experimento, a área experimental estava sendo utilizada para a produção de silagem, com milho (Zea mays) no verão, e no inverno azevém (Lolium multiflorum), por aproximadamente 16 anos. A adubação 
utilizada no período era ELBL, complementado com fertilizantes minerais. Tais fatores foram determinantes para a elevada fertilidade encontrada no início do experimento.

Os tratamentos foram distribuídos em três blocos casualizados, em arranjo fatorial $3 \times 4$, contendo três níveis de adubação mineral $(0,50$ e $100 \%$ da dose recomendada para as culturas) e quatro níveis de adubação orgânica $(0,30,60$ e $90 \mathrm{~m}^{3} \mathrm{ha}^{-1}$ ano-1 $^{-1}$, totalizando 12 tratamentos. 0 tamanho de cada unidade experimental foi de $33,6 \mathrm{~m}^{2}(4,8 \times 7,0 \mathrm{~m})$.

Tabela 1. Características químicas do solo da área de estudo antes da instalação do experimento (1998) e em área sob condição nativa (2004).

\begin{tabular}{|c|c|c|c|c|c|c|c|c|c|}
\hline Profundidade & $\begin{array}{c}\mathrm{pH} \\
\mathrm{CaCl}_{2}\end{array}$ & $\mathrm{H}^{+}+\mathrm{Al}^{3+}$ & $\mathrm{Al}^{3+}$ & $\mathrm{K}^{+}$ & $\mathrm{Ca}^{2+}$ & $\mathrm{Mg}^{2+}$ & $\begin{array}{c}P \\
\text { Mehlich } 1\end{array}$ & СОT & V \\
\hline & \multicolumn{9}{|c|}{ Área experimental } \\
\hline $\mathrm{m}$ & & & ----.. & nol $d r$ & ------- & & $\mathrm{mg} \mathrm{dm}^{-3}$ & $\mathrm{~g} \mathrm{dm}^{-3}$ & $\%$ \\
\hline $0-0,10$ & 5,1 & 6,09 & 0,08 & 0,32 & 3,75 & 1,67 & 15,6 & 26,0 & 48,5 \\
\hline \multirow[t]{2}{*}{$0,10-0,30$} & 5,3 & 4,97 & 0,03 & 0,15 & 3,99 & 1,86 & 7,1 & 21,0 & 54,7 \\
\hline & \multicolumn{9}{|c|}{ Área nativa } \\
\hline $0-0,05$ & 4,2 & 9,7 & 2,7 & 0,21 & 0,50 & 0,15 & 8,2 & 49,8 & 8,1 \\
\hline $0,05-0,10$ & 4,1 & 10,5 & 3,4 & 0,10 & 0,10 & 0,01 & 3,5 & 38,9 & 1,9 \\
\hline $0,10-0,30$ & 4,2 & 9,0 & 3,3 & 0,07 & 0,01 & 0,0 & 2,3 & 36,5 & 0,9 \\
\hline $0,30-0,50$ & 4,3 & 6,7 & 2,6 & 0,04 & 0,0 & 0,0 & 1,5 & 26,9 & 0,6 \\
\hline $0,50-0,80$ & 4,4 & 7,2 & 2,3 & 0,04 & 0,0 & 0,0 & 2,8 & 23,2 & 0,6 \\
\hline
\end{tabular}

O experimento foi conduzido em SPD durante seis anos agrícolas (1997-2003), sendo utilizada a rotação de culturas soja (FT Abyara)/ aveia preta (Comum)/milho (AG 9012 em 1998 e DKB 214 em 2001)/trigo (OR1)/ feijão (carioca comum)/aveia branca (Orla). As aveias e o trigo foram cultivados no inverno, e as demais no verão, portanto, foram utilizadas no experimento duas safras de cada cultura. Os espaçamentos entre fileiras foram de 0,$8 ; 0,4 ; 0,4$ e 0,17 $\mathrm{m}$ para milho, feijão, soja e culturas de inverno (trigo e aveias), respectivamente.

A adubação mineral foi baseada na análise inicial do solo, e a quantidade de adubo mineral correspondente a $100 \%$ do recomendado para as culturas, sem aplicação de esterco, totalizou após seis anos de cultivo em 484,340 e $340 \mathrm{~kg} \mathrm{ha}^{-1}$ de $\mathrm{N}, \mathrm{P}_{2} \mathrm{O}_{5}$ e $\mathrm{K}_{2} \mathrm{O}$.Para o fornecimento dos nutrientes foram utilizados fertilizantes mistos (NPK) contendo fontes solúveis de nutrientes. A adubação de semeadura foi feita em sulcos, depositando o adubo a aproximadamente $5 \mathrm{~cm}$ abaixo da semente no milho, soja e feijão e próximo à semente no trigo. A adubação de cobertura foi aplicada a lanço em todos os tratamentos nas doses (considerando o nível de $100 \%$ de adubação mineral) de 45,90 e $45 \mathrm{~kg} \mathrm{ha}^{-1}$ de $\mathrm{N}$, para trigo, milho e feijão, respectivamente, entre os estádios das culturas de V2 e V4, utilizando a uréia como fonte. Nas aveias, utilizadas com objetivo de cobertura verde, não foi aplicado adubo mineral de semeadura e de cobertura e na soja, não foi feita adubação nitrogenada de cobertura.

O esterco utilizado foi proveniente da criação de bovinos leiteiros em sistema de confinamento (Free stall), sendo composto pela mistura de fezes e urina, além de outros materiais provenientes do processo de criação, como água de lavagem e resto de alimentos. A dose de esterco foi aplicada em duas parcelas iguais, uma antes da semeadura das culturas de verão e outra antes da semeadura de inverno, sendo que o esterco foi distribuído manualmente na superfície com auxílio de um regador, sem incorporação. As características do esterco de três amostras coletadas no período do experimento foram: $78 \mathrm{~g} \mathrm{~kg}^{-1}$ de matéria seca, 20 $\mathrm{g} \mathrm{kg}^{-1}$ de $\mathrm{N}, 9,1 \mathrm{~g} \mathrm{~kg}^{-1}$ de $\mathrm{P}, 40 \mathrm{~g} \mathrm{~kg}^{-1}$ de $\mathrm{K}, 21 \mathrm{~g} \mathrm{~kg}^{-1}$ de $\mathrm{Ca}, 13 \mathrm{~g} \mathrm{~kg}^{-1}$ de $\mathrm{Mg}, 2600 \mathrm{mg} \mathrm{kg}^{-1}$ de Fe, 510 $\mathrm{mg} \mathrm{kg}^{-1}$ de $\mathrm{Zn}$ e $105 \mathrm{mg} \mathrm{kg}^{-1}$ de Cu.

Transcorridos seis anos agrícolas a coleta do solo foi realizada em cinco profundidades $(0-0,05, \quad 0,05-0,10,0,10-0,30,0,30-0,50$ e 0,50$0,80 \mathrm{~m})$; nas profundidades de $0-0,05,0,05-0,10$ e $0,10-0,30 \mathrm{~m}$, foram retiradas 12 subamostras simples para compor uma amostra composta, utilizando trado calador. Nas profundidades 0,30-0,50 e 0,50-0,80 m, cada amostra composta foi determinada a partir de 6 subamostras 
simples, com trado holandês. A amostragem foi realizada aleatoriamente nas parcelas e com cuidado para a não contaminação das amostras superiores.

Nas amostras foram determinados $\mathrm{pH}$ em $\mathrm{CaCl}_{2} 0,01 \mathrm{~mol} \mathrm{~L}^{-1}$ (relação solo:solução 1:2,5); a acidez potencial $\left(\mathrm{H}^{+}+\mathrm{Al}^{3+}\right)$ estimada pelo método do pH SMP; condutividade elétrica (CE) no extrato de saturação; os teores trocáveis de $\mathrm{Al}\left(\mathrm{Al}^{3+}\right), \mathrm{Ca}\left(\mathrm{Ca}^{2+}\right)$ e $\mathrm{Mg}\left(\mathrm{Mg}^{2+}\right)$, extrator $\mathrm{KCl} 1 \mathrm{~mol} \mathrm{~L}^{-1} ; \mathrm{P}$ e $\mathrm{K}^{+}$extrator Mehlich ${ }^{-1}$; segundo métodos descritos em EMBRAPA (2011). Com esses resultados foram calculados os valores de saturação por bases (V) e a capacidade de troca de cátions $\left(\mathrm{CTC}_{\mathrm{pH} 7,0}\right)$ e a relação $\mathrm{Ca}^{2+} / \mathrm{Mg}^{2+}$. A determinação do carbono orgânico total (COT) foi realizada de acordo com o método colorimétrico de Raije \& Quaggio (1983). Os micronutrientes foram determinados utilizando $O$ método de extração com água quente para $B$ (Silva, 1999), Cl (Tedesco et al., 1995), e o extrator

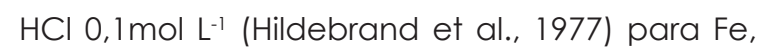
Cu, Zn e Mn. Espectrofotômetro de absorção atômica foi utilizado na determinação de $\mathrm{Ca}^{2+}, \mathrm{Mg}^{2+}, \mathrm{Fe}, \mathrm{Cu}, \mathrm{Zn}$ e $\mathrm{Mn}$; espectrofotômetro de chama foi utilizado na determinação de $\mathrm{K}^{+}$e espectrofotômetro UV-VIS foi utilizado na determinação de $\mathrm{P}, \mathrm{B}$ e Cl.

Os dados de cada camada foram analisados isoladamente, sendo submetidos à análise de variância $(p<0,05)$. Quando foi significativa as médias dos tratamentos de adubação orgânica foram submetidos a análise por regressão e a adubação mineral foi submetida ao teste de Tukey $(p<0,05)$.

\section{Resultados e Discussão}

Transcorridos seis anos de manejo da adubação em SPD, foram registradas alterações nos valores dos seguintes atributos químicos: $\mathrm{pH}$, $\mathrm{H}^{+}+\mathrm{Al}^{3+}, \mathrm{Ca}^{2+}, \mathrm{Mg}^{2+}, \mathrm{Ca}^{2+} / \mathrm{Mg}^{2+}, \mathrm{K}^{+}, \mathrm{V}, \mathrm{CE}, \mathrm{P}, \mathrm{COT}, \mathrm{Zn}$ e Cu na adubação orgânica com ELBL e; de pH, $\mathrm{H}^{+}+\mathrm{Al}^{3+}, \mathrm{K}^{+}, \mathrm{CE}, \mathrm{P}, \mathrm{Zn}$ e Cu na adubação mineral. Contudo, não ocorreu interação entre os fatores estudados (Tabela 2).

A adubação mineral provocou redução do $\mathrm{pH}(0,05-0,10$ e 0,10-0,30 m) e elevação de $\mathrm{H}^{+}+\mathrm{Al}^{3+}(0-0,05$ e 0,05-0,10 m) (Tabela 3). Esses resultados confirmam que a frente de acidificação decorrente do cultivo e da adubação das culturas ocorre das camadas superiores para as inferiores (Soratto \& Crusciol, 2008). Por outro lado, a aplicação de ELBL por seis anos propiciou a manutenção do $\mathrm{pH}$ e da acidez potencial do solo nas duas primeiras camadas (Figura 1). Corroborando com resultados aqui obtidos, o efeito do uso de esterco na manutenção do pH do solo tem sido reportado em experimentos de curto (Assmann et al., 2007) e longo prazo (Vitosh et al., 1973; Silva et al., 2008).

Tabela 2. Resumo da análise de variância para atributos químicos do solo nas camadas de 0-0,05, 0,05-0,10, 0, 100,30, 0,30-0,50 e 0,50-0,80 m de profundidade, após seis anos de adubação orgânica e mineral.

\begin{tabular}{|c|c|c|c|c|c|c|c|c|c|c|c|c|c|c|c|c|c|}
\hline Prof. & $\begin{array}{l}\text { Fonte de } \\
\text { variação }\end{array}$ & $\mathrm{pH}$ & $\mathrm{H}+\mathrm{Al}$ & $\mathrm{Ca}$ & $\mathrm{Mg}$ & $\mathrm{Ca} / \mathrm{Mg}$ & K & V & CE & $P$ & СОT & B & $\mathrm{Cl}$ & $\mathrm{Fe}$ & $\mathrm{Zn}$ & $\mathrm{Cu}$ & $M n$ \\
\hline$m$ & & & & & & & & & & & & & & & & & \\
\hline \multirow[t]{3}{*}{$0-0,05$} & 0 & $\mathrm{~L}^{*}$ & $L^{* *}$ & $L^{*}$ & $L^{* *}$ & $L^{* *}$ & $\mathrm{~L}^{* *}$ & $\mathrm{~L}^{* *}$ & $L^{*}$ & $\mathrm{~L}^{*}$ & ns & $\mathrm{ns}$ & $\mathrm{ns}$ & $\mathrm{ns}$ & $\mathrm{L}^{* *}$ & $\mathrm{~L}^{* *}$ & $\mathrm{~ns}$ \\
\hline & $M$ & ns & $*$ & ns & ns & ns & ns & ns & ns & $*$ & ns & $\mathrm{ns}$ & $\mathrm{ns}$ & $\mathrm{ns}$ & $*$ & $*$ & ns \\
\hline & $O * M$ & $\mathrm{~ns}$ & ns & ns & ns & ns & ns & ns & ns & ns & ns & ns & ns & $\mathrm{ns}$ & ns & ns & $\mathrm{ns}$ \\
\hline \multirow[t]{3}{*}{$0,05-0,10$} & 0 & $\mathrm{~L}^{*}$ & $\mathrm{~L}^{* *}$ & ns & ns & $L^{* *}$ & $\mathrm{~L}^{* *}$ & $\mathrm{~L}^{* *}$ & $\mathrm{~L}^{* *}$ & ns & $\mathrm{L}^{* *}$ & ns & ns & $\mathrm{ns}$ & $\mathrm{ns}$ & ns & $\mathrm{ns}$ \\
\hline & $M$ & $*$ & $*$ & ns & ns & ns & $*$ & ns & ns & ns & ns & ns & ns & ns & $*$ & $*$ & ns \\
\hline & $O^{*} M$ & ns & ns & ns & ns & ns & ns & ns & ns & ns & ns & $\mathrm{ns}$ & $\mathrm{ns}$ & $\mathrm{ns}$ & ns & ns & ns \\
\hline \multirow[t]{3}{*}{$0,10-0,30$} & 0 & $\mathrm{~ns}$ & ns & ns & ns & ns & $\mathrm{L}^{* *}$ & ns & $\mathrm{L}^{* *}$ & $\mathrm{~ns}$ & ns & $\mathrm{ns}$ & $\mathrm{ns}$ & $\mathrm{ns}$ & $\mathrm{ns}$ & ns & ns \\
\hline & M & $*$ & ns & ns & ns & ns & $*$ & ns & $*$ & ns & ns & ns & ns & ns & ns & ns & ns \\
\hline & $O^{*} M$ & ns & ns & ns & ns & ns & ns & $\mathrm{ns}$ & ns & ns & ns & ns & ns & $\mathrm{ns}$ & ns & ns & ns \\
\hline \multirow[t]{3}{*}{$0,30-0,50$} & O & $\mathrm{ns}$ & ns & ns & ns & $\mathrm{ns}$ & $\mathrm{L}^{* *}$ & ns & $\mathrm{L}^{* *}$ & ns & ns & ns & ns & $\mathrm{ns}$ & $\mathrm{ns}$ & ns & ns \\
\hline & $M$ & $\mathrm{~ns}$ & $\mathrm{~ns}$ & ns & ns & ns & $*$ & ns & ns & ns & ns & ns & ns & ns & ns & ns & ns \\
\hline & $O * M$ & ns & ns & ns & ns & ns & ns & ns & ns & ns & ns & $\mathrm{ns}$ & $\mathrm{ns}$ & $\mathrm{ns}$ & ns & ns & ns \\
\hline \multirow[t]{3}{*}{$0,50-0,80$} & 0 & $\mathrm{~ns}$ & ns & ns & ns & $\mathrm{ns}$ & $\mathrm{L}^{* *}$ & ns & $\mathrm{L}^{* *}$ & $\mathrm{~ns}$ & ns & ns & ns & $\mathrm{ns}$ & $\mathrm{ns}$ & $\mathrm{ns}$ & ns \\
\hline & $M$ & ns & ns & ns & ns & ns & ns & ns & ns & ns & ns & ns & ns & ns & ns & ns & ns \\
\hline & $O^{*} M$ & $\mathrm{~ns}$ & ns & ns & ns & ns & ns & ns & ns & ns & ns & ns & ns & $\mathrm{ns}$ & ns & ns & ns \\
\hline
\end{tabular}


Tabela 3. Efeito da adubação mineral nos atributos químicos $\mathrm{pH}, \mathrm{H}^{+}+\mathrm{Al}^{3+}, \mathrm{K}^{+}, \mathrm{CE}, \mathrm{P}, \mathrm{Zn}$ e Cu.

\begin{tabular}{cccccccc}
\hline \multirow{2}{*}{ Adubação } & \multicolumn{2}{c}{$\mathrm{pH} \mathrm{H}^{+}+\mathrm{Al}^{\mid+}$} & \multicolumn{3}{c}{$\mathrm{K}^{+}$} \\
\cline { 2 - 8 } & $0,05-0,10$ & $0,10-0,30$ & $0-0,05$ & $0,05-0,10$ & $0,05-0,10$ & $0,10-0,30$ & $0,30-0,50$ \\
\hline $0 \%$ & $5,3 \mathrm{a}$ & $5,4 \mathrm{a}$ & $4,1 \mathrm{~b}$ & $5,0 \mathrm{~b}$ & $0,43 \mathrm{~b}$ & $0,32 \mathrm{~b}$ & $0,25 \mathrm{~b}$ \\
$50 \%$ & $5,1 \mathrm{ab}$ & $5,1 \mathrm{~b}$ & $4,7 \mathrm{a}$ & $5,5 \mathrm{a}$ & $0,47 \mathrm{ab}$ & $0,38 \mathrm{a}$ & $0,26 \mathrm{~b}$ \\
$100 \%$ & $5,0 \mathrm{~b}$ & $5,2 \mathrm{ab}$ & $4,8 \mathrm{a}$ & $5,6 \mathrm{a}$ & $0,53 \mathrm{a}$ & $0,42 \mathrm{a}$ & $0,36 \mathrm{a}$ \\
\hline \multirow{2}{*}{ Adubação } & $\mathrm{CE}$ & $\mathrm{P}$ & \multicolumn{5}{c}{$\mathrm{Zn} \mathrm{Cu}$} \\
\cline { 2 - 8 } & $0,10-0,30$ & $0-0,05$ & $0-0,05$ & $0,05-0,10$ & $0-0,05$ & $0,05-0,10$ \\
\hline $0 \%$ & $75 \mathrm{~b}$ & $29 \mathrm{~b}$ & $9 \mathrm{ab}$ & $3 \mathrm{~b}$ & $1,74 \mathrm{ab}$ & $1,49 \mathrm{~b}$ \\
$50 \%$ & $82 \mathrm{a}$ & $42 \mathrm{ab}$ & $5 \mathrm{~b}$ & $4 \mathrm{~b}$ & $1,67 \mathrm{~b}$ & $1,37 \mathrm{~b}$ \\
$100 \%$ & $83 \mathrm{a}$ & $49 \mathrm{a}$ & $10 \mathrm{a}$ & $8 \mathrm{a}$ & $1,86 \mathrm{a}$ & $1,75 \mathrm{a}$ \\
\hline Letras seguidas pela mesma letra não diferem entre si pelo teste de Tukey (p<0,05).
\end{tabular}
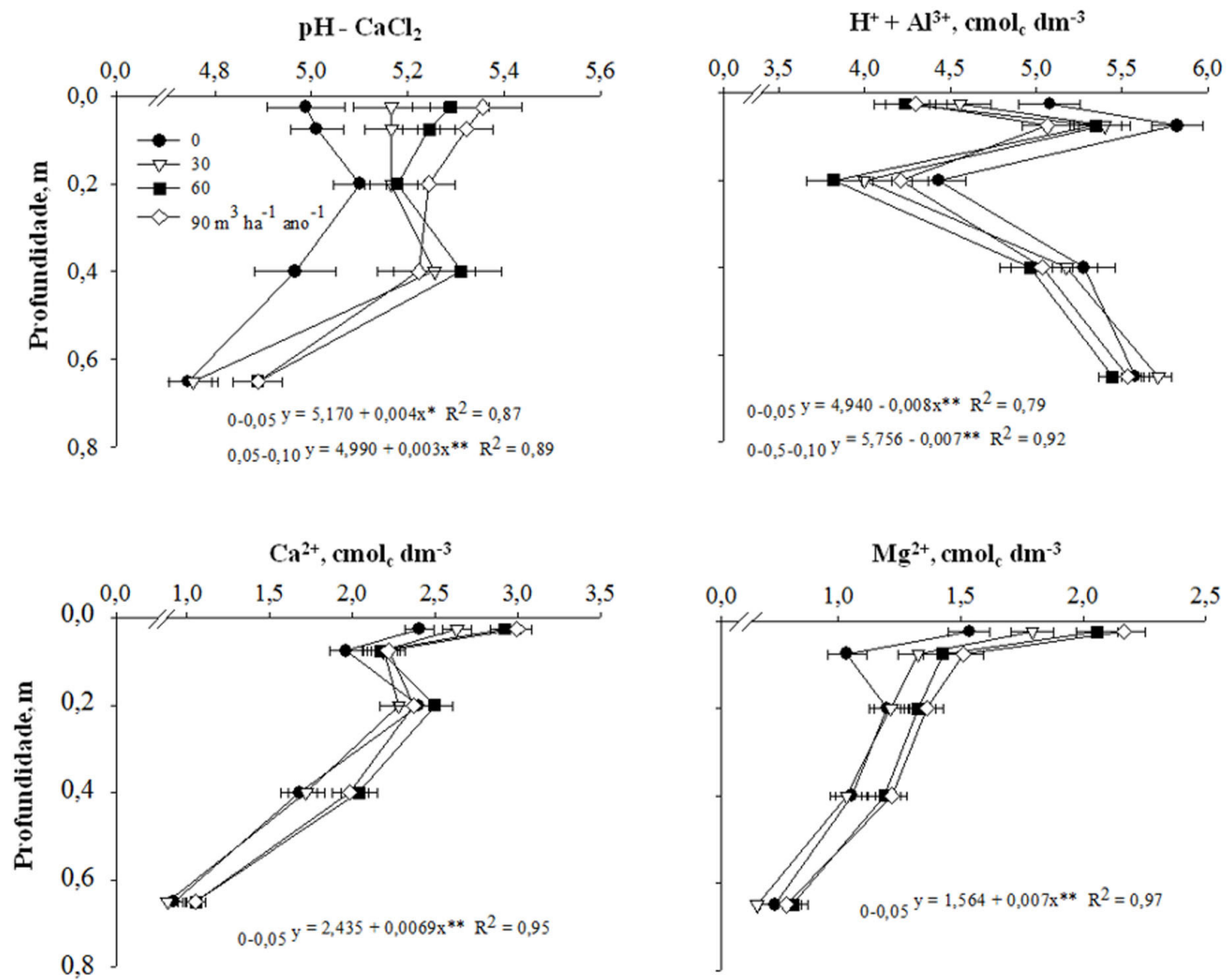

Figura 1. $\mathrm{pH}\left(\mathrm{CaCl}_{2}\right)$, acidez potencial $\left(\mathrm{H}^{+}+\mathrm{Al}^{3+}\right), \mathrm{Ca}^{2+}$ e $\mathrm{Mg}^{2+}$ trocáveis no solo decorrente da aplicação de doses de ELBL. * $\mathrm{e}^{* *}$ significativo a $5 \%$ e a $1 \%$, respectivamente. Barras horizontais representam desvio padrão das médias.

O solo da área experimental no início do estudo apresentava 0,08 e $0,03 \mathrm{cmol}_{\mathrm{c}} \mathrm{dm}^{-3}$ de $\mathrm{Al}^{3+}$, nas camadas de $0-0,10$ e 0,10-0,30 m, respectivamente (Tabela 1). Condizente com os resultados de $\mathrm{pH}$ do solo, transcorridos seis anos de manejo da adubação mineral e orgânica na área, não foi detectado presença de Al para nenhuma situação, exceto na camada de 0,50 $0,80 \mathrm{~m}$, com teor médio de $0,09 \mathrm{cmol}_{\mathrm{c}} \mathrm{dm}^{-3}$, sem efeito significativo para os fatores de adubação avaliados (dados não apresentados).
Os teores de Ca e Mg trocáveis $\left(\mathrm{Ca}^{2+} \mathrm{e}\right.$ $\mathrm{Mg}^{2+}$ ) tiveram incrementos apenas na camada mais superficial do solo (0-0,05 m), com ajuste linear em função das doses de esterco (Figura 1). Esses incrementos devem se a adição por seis anos consecutivos desses nutrientes com o ELBL (21 $\mathrm{g} \mathrm{kg}^{-1}$ de Ca e $13 \mathrm{~g} \mathrm{~kg}^{-1}$ de $\mathrm{Mg}$ ) e a baixa influência da exportação pelas culturas. Analisando a distribuição de $\mathrm{Ca}^{2+}$ no perfil do solo, verifica-se que na camada de 0,05-0,10 $\mathrm{m} \circ$ teor do nutriente foi menor em relação 
à camada sobrejacente, sendo que na camada de 0,10-0,30 m os valores voltaram a aumentar. $\bigcirc \mathrm{Mg}^{2+}$ foi maior na primeira camada com decréscimo nas camadas subjacentes, contudo, sem aplicação de esterco verificouse comportamento semelhante ao $\mathrm{Ca}^{2+}$ na camada de 0,05-0,10 m.

Em geral, O Ca e o Mg apresentam estratificação no perfil do solo sob SPD, com teores que decrescem da camada superficial para as camadas inferiores (Arf et al., 2014). A distribuição irregular, com menor teor na camada de 0,05-0,10 $\mathrm{m}$ tem se repetido em alguns trabalhos conduzidos sob SPD, todavia, sem uma resposta clara para este fenômeno (Duiker\&Beegle, 2006; Scherer et al., 2007). Entretanto, a camada de 0,05-0,10 m (com redução dos teores de $\mathrm{Ca}^{2+}$ ) foi a mesma em que a acidez potencial solo foi maior (Figura 1), por isso a variação do $\mathrm{Ca}^{2+}$ pode estar relacionada ao aumento da acidez potencial. Esse aumento não decorreu dá adubação, pois ocorreu no tratamento sem adubação. Além disso, não é o padrão do Latossolo na condição de área nativa (Tabela 1). Assim, variações nos atributos físicos (densidade, porosidade) e biológicos (microorganismos e raízes) inerentes ao sistema de uso e manejo do solo provavelmente estejam afetando a distribuição da acidez do solo.

A relação $\mathrm{Ca}^{2+} / \mathrm{Mg}^{2+}$ teve redução linear com as doses de esterco nas camadas de 0-0,05 e 0,05-0, 10 m (Figura 2). Em nenhuma das condições de adubação orgânica e adubação mineral os valores da relação $\mathrm{Ca}^{2+} / \mathrm{Mg}^{2+}$ atingiram níveis considerados inadequados. Neto et al. (2011) reportam que relações maiores que 5/1 afetam negativamente a produção de milho, sendo que a alta produtividade foi alcançada em relações baixas (<3). Assim, a redução da relação $\mathrm{Ca}^{2+} / \mathrm{Mg}^{2+}$ devido a aplicação de ELBL pode ser considerada benéfica.
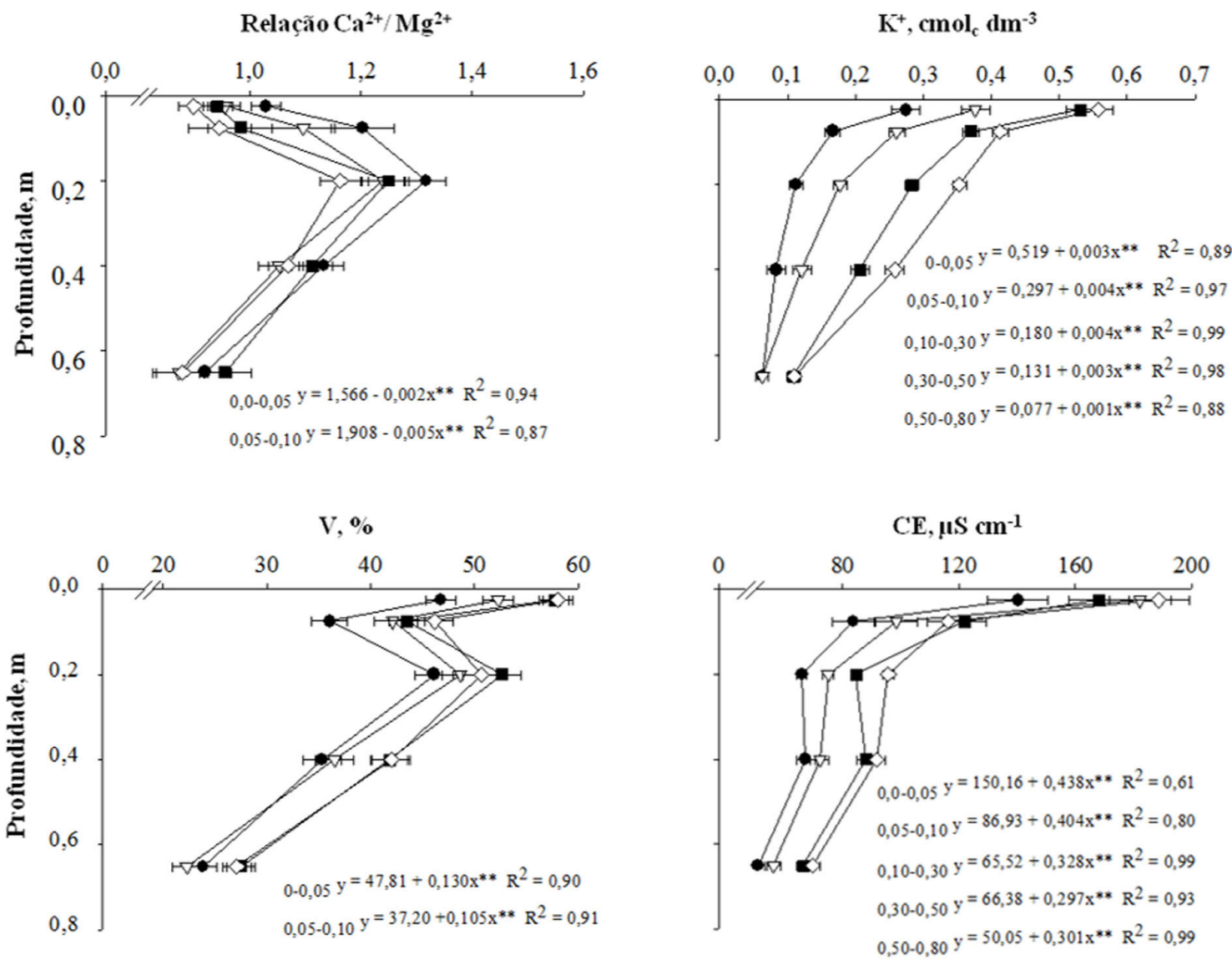

Figura 2. Relação $\mathrm{Ca}^{2+} / \mathrm{Mg}^{2+}, \mathrm{K}^{+}$, saturação por bases (V) e condutividade elétrica (CE) no solo decorrente da aplicação de doses de ELBL. ${ }^{*} \mathrm{e}^{* *}$ : significativo a $5 \%$ e a $1 \%$, respectivamente. Barras horizontais representam desvio padrão das médias. 
Os teores de $\mathrm{K}^{+}$no solo aumentaram consideravelmente com a aplicação de ELBL, sobretudo nas maiores doses, sendo que este efeito foi verificado tanto em superfície como em sub-superfície (Figura 2). Por sua vez, a adubação mineral elevou os teores de $\mathrm{K}^{+}$no solo em três camadas $(0,05-0,10 ; 0,10-0,30 ; 0,30-0,50)$ (Tabela 3). Esse comportamento indica que a adição de $\mathrm{K}^{+}$ao solo (via adubação orgânica ou mineral) é superior a perda por exportação junto à colheita das culturas produtoras de grãos. Estudando os atributos químicos de solos sob diferentes manejos de adubação por longos períodos (15-20 anos), Scherer et al. (2010) verificaram aumentos nos teores de $\mathrm{K}^{+}$ com adubação mineral e adubação orgânica. Nesse estudo, a adubação mineral aumentou - $\mathrm{K}^{+}$apenas nas camadas mais superficiais, enquanto que a adubação orgânica aumentou os teores do nutriente até $1 \mathrm{~m}$ de profundidade. Todavia, quando a produção vegetal é utilizada em sua totalidade, como para a produção de silagem, não é comum ser registrado excesso de $\mathrm{K}^{+}$no solo (Vitosh et al., 1973; Silva et al., 2010).

A saturação por bases (V) do solo foi elevada pela aplicação de ELBL nas camadas 0-0,05 e 0,05-0,10 m (Figura 2). Esses resultados foram condizentes com o acréscimo nos teores de $\mathrm{Ca}^{2+}, \mathrm{Mg}^{2+}$ e $\mathrm{K}^{+}$proporcionado pela aplicação consecutiva de ELBL, evidenciando o potencial da adubação orgânica com dejetos em elevar os teores de vários nutrientes simultaneamente.

Os valores de CE foram influenciados pelas doses de ELBL de maneira similar aos teores de $\mathrm{K}^{+}(0$ até $0,80 \mathrm{~m})$, enquanto a adubação mineral variou apenas na camada de 0,10-0,30 m (Figura 2; Tabela 3). A ascensão nos valores de CE sob adubação orgânica com o uso de estercos é atribuído, principalmente, aos íons sódio $\left(\mathrm{Na}^{+}\right)$e $\mathrm{K}^{+}$(Josan et al., 2005), nitrato $\left(\mathrm{NO}_{3}^{-}\right)$(Jokela, 1992), sulfato $\left(\mathrm{SO}_{4}^{-}\right)$e $\mathrm{Cl}^{-}$(Chang et al., 1990). Embora a CE tenha aumentado, principalmente sob as maiores doses de ELBL e $100 \%$ da adubação mineral, tais resultados corroboram com valores apresentados por Chang et al. (1990), sendo considerados como teores médios.

Em relação ao $P$, se constatou ascendência nos teores na camada de 0-0,05 m com a aplicação de ELBL e com a adubação mineral (Figura 3). A adubação mineral aumentou os teores de $\mathrm{P}$ de maneira isolada do esterco, o que decorre da aplicação de $340 \mathrm{~kg} \mathrm{ha}^{-1}$ de $\mathrm{P}_{2} \mathrm{O}_{5}$ em seis anos (equivalente ao nível de $100 \%$ ) de manejo da adubação. 0 solo da área experimental é classificado como um Latossolo Bruno distrófico típico, no qual predominam argilas dos tipos caulinita, gibbsita, goethita e hematita (Martins, 2002), que, por apresentar alto grau de adsorção específica com o P, provocam o acúmulo do nutriente em superfície (Vilar et al., 2010). Além disso, o fator manejo do solo também contribuiu para que isso ocorresse, tendo em vista que no SPD o solo não é submetido ao revolvimento intensivo. Contudo, o enriquecimento da camada superficial deve ser monitorado com cautela, tendo em vista que podem ocorrer perdas de solo e P em situações favoráveis ao escoamento superficial, tendo potencial para diminuir a qualidade da água nos cursos a jusante (Mori et al., 2009).

Os teores de COT foram influenciados pela aplicação de ELBL apenas na camada de 0,05-0,10 m (Figura 3), enquanto que a adubação mineral não alterou o COT (Tabela 2). Embora o aumento nos teores de COT não seja perceptível tão rápido quanto os teores de nutrientes ou CE, a adubação orgânica por vários anos consecutivos tem demonstrado aumento do COT, principalmente na superfície do solo (Scherer et al., 2007; Silva et al., 2008). Em termos de distribuição no perfil analisado (0-0,80 m), os maiores teores de COT ocorrem na camada superficial $(0-0,05 \mathrm{~m})$, diminuindo gradativamente em profundidade, tendência em solo sob SPD, resultado está associado ao manejo do solo sem o revolvimento (Nunes et al., 2011)

Os micronutrientes $\mathrm{B}$ e $\mathrm{Cl}$ não apresentaram nenhuma relação significativa com a adição de ELBL após seis anos de aplicação, tão pouco influência da adubação mineral (Figura 3). Esse comportamento devese a baixa capacidade de adsorção de ambos os elementos no solo e a elevada precipitação pluviométrica média da região. Embora tenha sido observada a ocorrência de adsorção específica do B em alguns minerais do solo, sua 
elevada mobilidade e perda tem sido observada em diferentes condições (Rosolem \& Bíscaro, 2007; Oliveira Neto et al., 2009). A interação do $\mathrm{Cl}$ com minerais do solo é inexpressiva, o que o torna altamente disponível na solução do solo, assim, é facilmente transportado com a água que infiltra no solo (Pessoa et al., 2010).

\section{P-Melhlich1, mg dm-3}

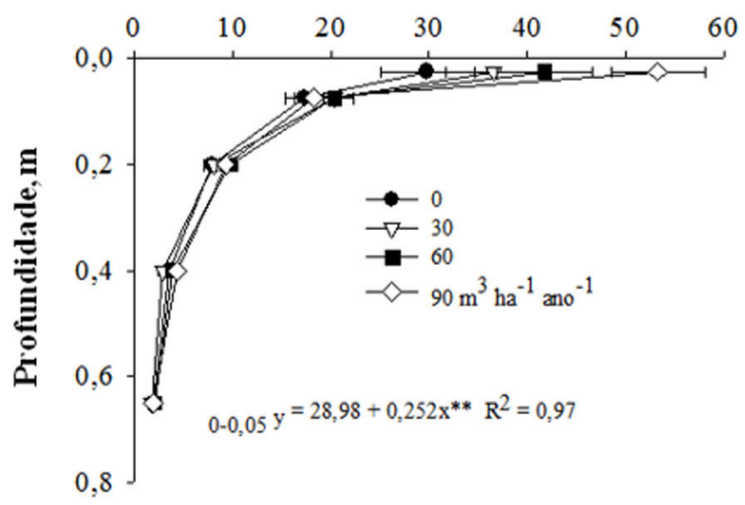

B, $\mathbf{m g} \mathbf{d m}^{-3}$

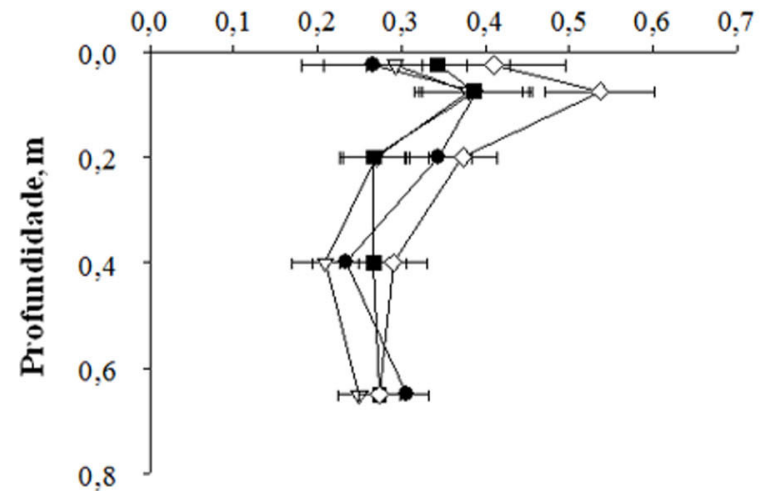

\section{COT, g kg-1 $^{-1}$}

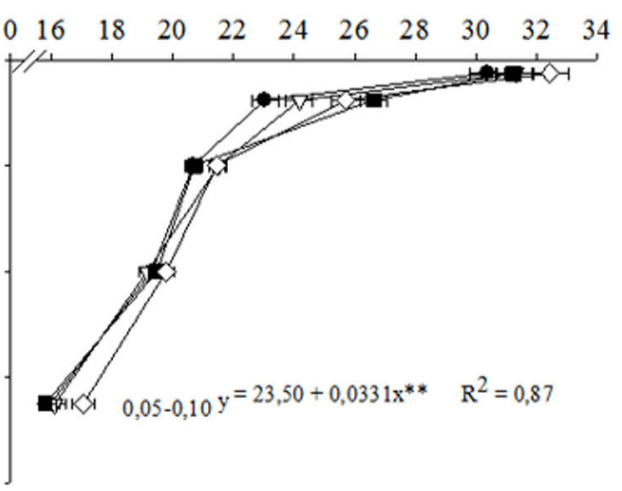

Cl, mg dm dm $^{-3}$

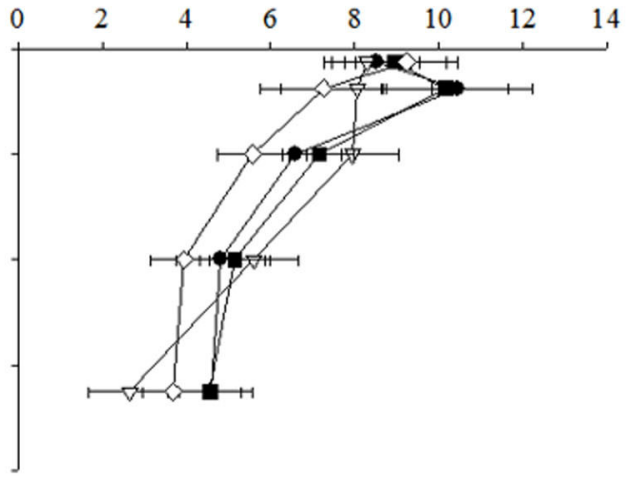

Figura 3. Fósforo (P), carbono orgânico total (COT), boro (B) e cloro (Cl) no solo decorrente da aplicação de doses de ELBL. * e **: significativo a $5 \%$ e a $1 \%$, respectivamente. Barras horizontais representam desvio padrão das médias.

Na camada de 0-0,05 $\mathrm{m}$ as variações médias dos micronutrientes catiônicos foram de 7,3-9,3 $\mathrm{mg} \mathrm{kg}^{-1}$ para Fe, 3-11,7 $\mathrm{mg} \mathrm{kg}^{-1}$ para Zn, 1,3-2,1 $\mathrm{mg} \mathrm{kg}^{-1}$ para Cu, 13,4-15,1 $\mathrm{mg} \mathrm{kg}^{-1}$ para Mn. Entretanto, apenas $\mathrm{Zn}$ e Cu tiveram comportamentos distintos em relação a adubação orgânica e mineral e, a variabilidade vertical no solo (Figura 4).

O incremento na dose de esterco elevou linearmente o teor de $\mathrm{Zn}$ e Cu na camada de 0-0,05 m. Porém, na adubação mineral ocorreu efeito significativo para as camadas de 0-0,05 e 0,0-0,10 m, sendo que os teores de Zn e Cu foram superiores no nível de 100\% de adubação. Esse acúmulo superficial tem sido evidenciado em solos sob SPD com a aplicação de dejetos de animais e em alguns casos com a adubação mineral, similarmente ao que ocorre com os teores de P no solo (Mattias et al., 2010; Scherer et al., 2010). Porém, vale ser destacado que a adubação orgânica em Latossolos (como no presente estudo com ELBL) proporciona menor alteração nos teores disponíveis em comparação aos Neossolos. Devido a composição mineralógica, os Latossolos são capazes de fixar maiores quantidades de $\mathrm{Zn}$ e Cu, o que torna os teores disponíveis menores (Scherer et al., 2010).

O acúmulo preferencial de Zn e Cu na camada mais superficial do solo é resultadodo manejo do solo em SPD e da forte interação dos elementos com os constituintes do solo, sendo que os minerais do solo retêm maior quantidade de Zn e a fração orgânica do solo retêm mais o Cu (Girotto et al., 2010). No caso do ELBL, o aumento dos teores de $\mathrm{Zn}$ e Cu devem-se ao esterco utilizado em nosso estudo apresentar 
teores médios de 510 e $105 \mathrm{mg} \mathrm{kg}^{-1}$, totalizando ao final de seis anos cerca de 7,2 e 1,5 $\mathrm{kg}$ de Zn e Cu adicionados ao solo a cada $30 \mathrm{~m}^{3} \mathrm{ha}$ 1 de dejeto, respectivamente. Já o efeito da adubação mineral nos teores de Zn e Cu no solo é menor, tendo em vista as baixas concentrações desses elementos nos fertilizantes mistos NPK [32,7 mg Zn kg-1 e 31,6 mg Cu kg-1] (Carvalho ef al., 2012), como os utilizados em nosso estudo.
Contudo, fatores inerentes ao sistema de manejo do solo e da adubação são importantes para compreender a dinâmica desses nutrientes no solo. A ciclagem de nutrientes, considerando que os resíduos culturais permaneceram sobre a superfície do solo devido ao manejo do solo ter sido em SPD, é um fator que pode ter contribuído para as alterações nos teores de $\mathrm{Zn}$ e Cu com a adubação mineral.

\section{$\mathrm{Fe}, \mathrm{mg} \mathrm{dm} \mathbf{d m}^{-3}$}

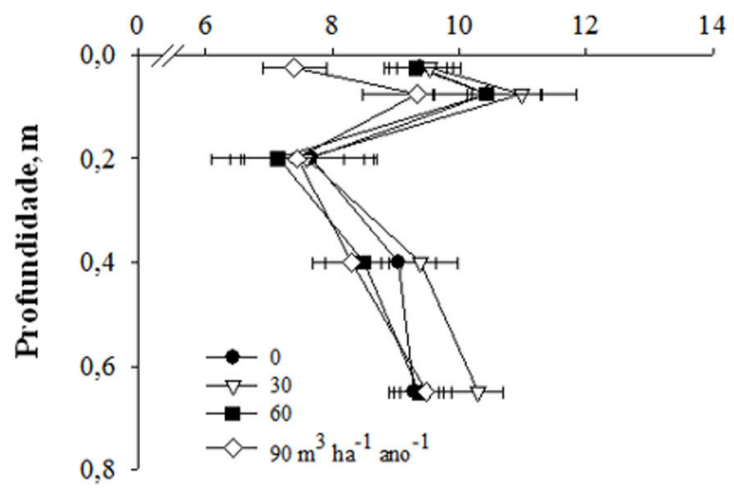

$\mathrm{Cu}, \mathrm{mg} \mathrm{dm}{ }^{-3}$

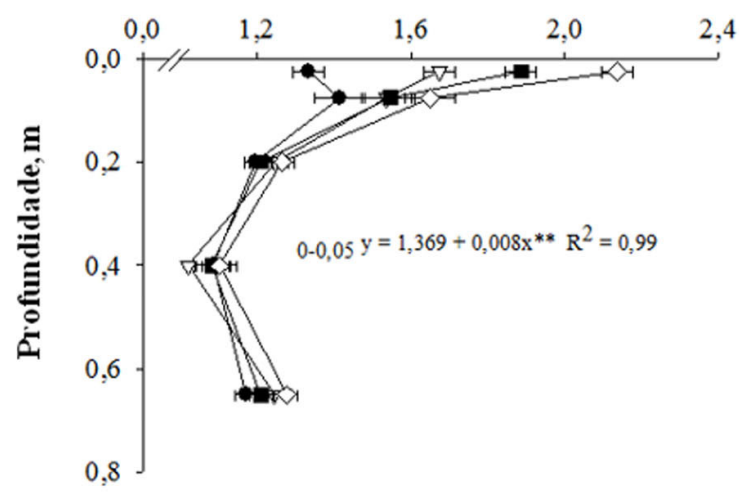

Zn, mg dm-3

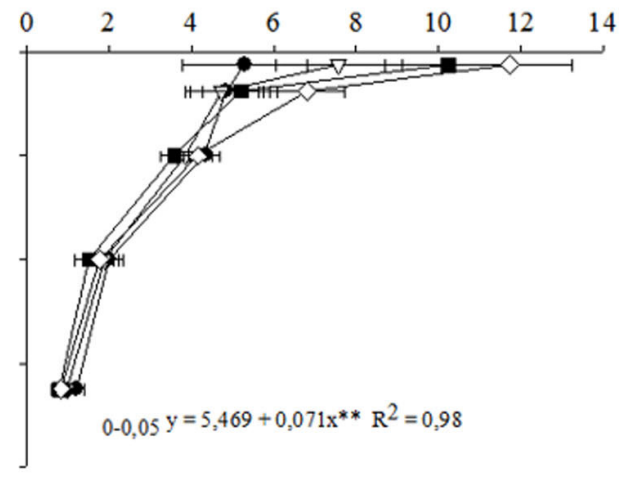

Mn, $\mathbf{m g ~ d m} \mathbf{d m}^{-\hat{3}}$

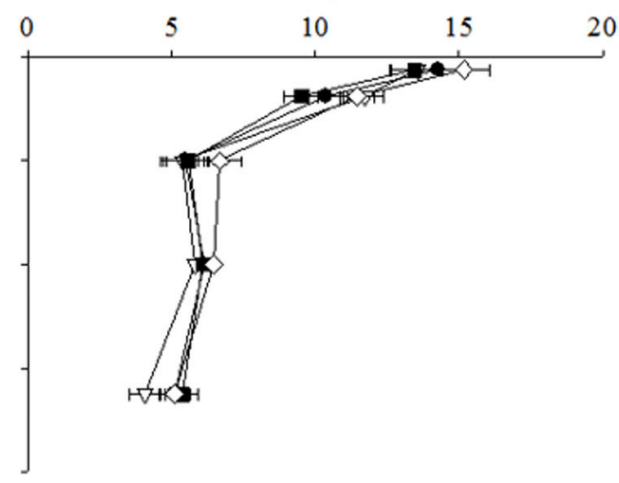

Figura 4. Ferro (Fe), zinco (Zn), cobre (Cu) e manganês (Mn) no solo decorrente da aplicação de doses de ELBL. * $e^{* *}$ : significativo a $5 \%$ e a $1 \%$, respectivamente. Barras horizontais representam desvio padrão das médias.

\section{Conclusões}

Transcorridos seis anos de manejo da adubação em Latossolo Bruno sob SPD, não houve alteração nos teores de $\mathrm{B}, \mathrm{Cl}$, Fe e $\mathrm{Mn}$ no solo. Por outro lado, a adubação mineral acidificou o solo, elevou os teores de $\mathrm{K}^{+}, \mathrm{P}$ e CE e, alterou os teores de $Z n$ e Cu. A adubação orgânica com ELBL foi fonte de nutrientes $\left(\mathrm{Ca}^{2+}\right.$, $\mathrm{Mg}^{2+}, \mathrm{K}^{+}, \mathrm{P}, \mathrm{Zn}$ e $\left.\mathrm{Cu}\right)$, elevou o COT e a CE e, manteve os atributos de acidez do solo $(\mathrm{pH}$, $\left.\mathrm{H}^{+}+\mathrm{Al}^{3+} \mathrm{eV}\right)$.

Em geral, a adubação orgânica com ELBL foi benéfica para a fertilidade do solo. Todavia, o acúmulo de $\mathrm{P}$ na camada de 0-0,05 m indica que é importante considerar com cautela o uso de altas doses de esterco, sobretudo em solos sob o risco de escoamento superficial. Os altos valores de $\mathrm{K}$ ao longo da camada de solo avaliada $(0-0,80 \mathrm{~m})$ indicam que nas maiores doses de ELBL houve perda de $\mathrm{K}^{+}$em profundidade.

\section{Referências}

Acosta, J.A.A.,Amado, T.J.C., Silva, L.S., Santi, A.,Weber, M.A. (2014). Decomposição da fitomassa de plantas de cobertura e liberação de nitrogênio em função da quantidade de 
resíduos aportada ao solo sob sistema plantio direto. Ciência Rural 44(5): 801-809.

Alvares, C.A., Stape, J.L., Sentelhas, P.C., Gonçalves, J.L.M., Sparovek, G. 2013. Köppen's climate classification map for Brazil. Meteorologis che Zeitschrift 22(6): 711-728.

Arf, O., Rodrigues, R.A.F., Nascente, A.S., Lacerda, M.C. 2014. Gesso aplicado na superfície do solo no desenvolvimento do arroz de terras altas sob plantio direto. Revista Brasileira de Engenharia Agrícola e Ambiental 18(11): 1136-1141.

Assmann, T.S., Assmann, J.M., Cassol, L.C., Diehl, R.Q., Manteli, C., Magiero, E.C. 2007. Desempenho da mistura forrageira de aveiapreta mais azevém e atributos químicos do solo em função da aplicação de esterco líquido de suínos. Revista Brasileira de Ciência do Solo 31: 515-1523.

Carvalho, V.G.B., Nascimento, C.W.A., Biondi, C.M. 2012. Potencial de fertilizantes e corretivos no aporte de micronutrientes ao solo. Revista Brasileira de Ciência do Solo 36: 931-938.

Ceretta, C.A., Brunetto, G.L., Girotto, E., Gatiboni, L.C., Lourenzi, C.R., Tiecher, T.L., Conti, L., Trentin, G., Miotto, A. 2010. Frações de fósforo no solo após sucessivas aplicações de dejetos de suínos em plantio direto. Pesquisa Agropecuária Brasileira 45(6): 593-602.

Chang, C., Sommerfeldt, T.G., Entz, T. 1990. Rates of soil chemical changes with eleven annual applications of cattle feedlot manure. Canadian Journal Soil Science 70: 673-681.

Conceição, P.C., Bayer, C., Dieckow, J., Santos, D.C. 2014. Fracionamento físico da matéria orgânica e índice de manejo de carbono de um Argissolo submetido a sistemas conservacionistas de manejo. Ciência Rural 44: 794-800.

Duiker, S.W., Beegle, D.B. 2006. Soil fertility distribuitions in long-term no-till, chisel/disk and moldboar plow/disk systems. Soil \& Tillage Research 88: 30-41.

Empresa Brasileira de Pesquisa Agropecuária - Embrapa. 2002. Caracterização dos solos do município de Castro. Embrapa Solos: Rio de Janeiro (Boletim de Pesquisa e Desenvolvimento, 09).

Empresa Brasileira de Pesquisa Agropecuária Embrapa. 2011. Manual de métodos de análise de solo. 2 ed. Embrapa, Rio de Janeiro, Brasil, 230 p.

Girotto, E., Ceretta, C.A., Brunetto, G., Santos, D.R., Silva, L.S., Lourenzi, C., Lorensini, F., Vieira, R.C.B., Schmatz, R. 2010. Acúmulo e formas de cobre e zinco no solo após aplicações sucessivas de dejeto líquido de suínos. Revista Brasileira de Ciência do Solo 34:955-965.

Hildebrand, C. 1977. Manual de análise química de solo e planta. Universidade Federal do Paraná, Curitiba, Brasil. 225 p.

Jokela, W.E. 1992. Nitrogen fertilizer and dairy manure effects on corn yield and soil nitrate. Soil Science Society American Journal 56: 148-154.

Josan, M.S., Nair, V.D., Herrera, D. 2005. Associated release of magnesium and phosphorus from active and abandoned dairy soils. Journal of Environmental Quality 34: 184-191.

Martins, R. 2002. Contribuição da reserva de potássio na nutrição e produção de trigo em solos do município de Castro, Estado do Paraná. 94 f. (Dissertação de Mestrado) - Universidade Federal do Paraná, Curitiba, Brasil.

Mattias, J.L., Ceretta, C.A., Nesi, C.N., Girotto, E., Trentin, E.E., Lourenzi, C.R., Vieira, R.C.B. 2010. Copper, zinc and manganese in soils of two watersheds in Santa Catarina with intensive use of pig slurry. Revista Brasileira de Ciência do Solo 34: 1445-1454.

Mori, H.F., Favaretto, N., Pauletti, V., Dieckow, J., Santos, W.L. 2009. Perda de água, solo e fósforo com aplicação de dejeto líquido bovino em Latossolo sob plantio direto e com chuva simulada. Revista Brasileira de Ciência do Solo 33: 189-198.

Neto, F.N., Roloff, G., Dieckow, J., Motta, A.C.V. 2011. Atributos de solo e cultura espacialmente distribuídos relacionados ao rendimento do milho. Revista Brasileira de Ciência do Solo 35(3): 1025-1036.

Nunes, R.F., Lopes, A.A.C., Sousa, D.M.G., Mendes, I.C. 2011 . Sistemas de manejo e os estoques de carbono e nitrogênio em latossolo de cerrado com a sucessão soja-milho. Revista Brasileira de Ciência do Solo 35(4): 1407-1419.

Oliveira Neto, W.D., Muniz, A.S., Silva, M.A.G., Castro, C., Borkert, C.M. 2009. Boron extraction and vertical mobility in Paraná state oxisol, Brazil. Revista Brasileira de Ciência do Solo 33: 1259 1267.

Pessoa, L.G.M., Oliveira, E.E.M., Freire, M.B.G.S., Freire, F.J., Miranda, M.A., Santos, R.L. 2010. Composição química e salinidade do lixiviado em dois solos cultivados com cebola irrigada com água salina. Revista Brasileira de Ciências Agrárias 5: 406-412.

Raij, B.V., Quaggio, J.A. 1983. Métodos de análise de solo. Instituto Agronômico, Campinas, Brasil. 31 p. Boletim Técnico, 81. 
Rosolem, C., Bíscaro, T. 2007. Adsorção e lixiviação de boro em Latossolo VermelhoAmarelo. Pesquisa Agropecuária Brasileira 42: 1473-1478.

Scherer, E.E., Baldissera, I.T., Nesi, C.N. 2007. Propriedades químicas de um Latossolo Vermelho sob plantio direto e adubação com esterco de suínos. Revista Brasileira de Ciência do Solo 31: 123-131.

Scherer, E.E., Nesi, C.N., Massoti, Z. 2010. Atributos químicos do solo influenciados por sucessivas aplicações de dejetos suínos em áreas agrícolas de Santa Catarina. Revista Brasileira de Ciência do Solo 34: 1375-1383.

Silva, J.C.P.M., Motta, A.C.V., Pauletti, V., Favaretto, N., Barcellos, M., Oliveira, A.S., Veloso, C., Costa e Silva, L.F. 2008. Esterco líquido de bovinos leiteiros combinado com adubação mineral sobre atributos químicos de um Latossolo Bruno. Revista Brasileira de Ciência do Solo 32: 2563-2572.

Silva, J.C.P.M., Motta, A.C.V., Pauletti, V., Veloso, C.M., Favaretto, N., Bastos, M., Oliveira, A.S., Costa e Silva, L.F. 2010. Esterco de gado leiteiro associado à adubação mineral e sua influência na fertilidade de um Latossolo sob plantio direto. Revista Brasileira de Ciência do Solo 34: 453-463.

Silva, F.C. 1999. Manual de Análises Químicas de Solos, Plantas e Fertilizantes. Embrapa, Brasília, Brasil, $370 \mathrm{p}$.

Soratto, R.P., Crusciol, C.A.C. 2008. Atributos químicos do solo decorrentes da aplicação em superfície de calcário e gesso em sistema plantio direto recém-implantado. Revista Brasileira de Ciência do Solo 32: 675-688.

Tedesco, M.J., Gianello, C., Bissani, C.A., Bohnen, H., Volkweiss, S.J. 1995. Análise de solo, Plantas e Outros Materiais. Universidade Federal do Rio Grande do Sul, Porto Alegre, Brasil, 174 p. (Boletim Técnico de Solos n. 5).

Vilar, C.C., Costa, A.C.S., Hoepers, A., Souza Júnior, I.G. 2010. Capacidade máxima de adsorção de fósforo relacionada a formas de ferro e alumínio em solos subtropicais. Revista Brasileira de Ciência do Solo 34: 1059-1068.

Vitosh, M.L., Davis, J.F., Knezek, B.D. 1973. Longterm effects of manure, fertilizer, and plow depth on chemical properties of soils and nutrient movement in a monoculture corn system. Journal of Environmental Quality 2: 296-299. 